\section{How old are distant stars?}

SIR-The existence of young stars at vast distances from the plane of our Galaxy, as discussed in a recent News and Views article $^{\prime}$, seems to be reasonably well understood for some of the varieties of star under investigation. In the case of the young, high-velocity A stars observed in the galactic halo, however, evidence is now available that the identification of those stars as blue stragglers is incorrect.

My new observations of a large sample of A stars at the South Galactic Pole, from near the galactic plane to around 10 kiloparsecs away from the plane $\mathrm{e}^{2,3}$, give detailed information on their kinematics, abun-

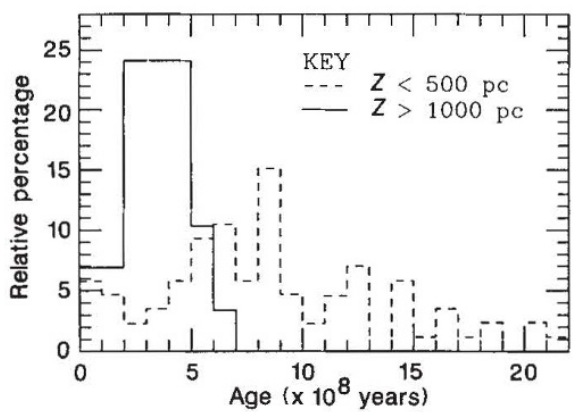

Fig. 1 The age distributions of two samples of young A stars at the South Galactic Pole, one group within 500 parsecs of the galactic plane, and the other at distances of 1-10 kiloparsecs away.

dances and, most importantly, their ages. The ages of main-sequence $\mathrm{A}$ stars may be derived using isochrones (same-ageevolution tracks) in colour-luminosity ( $\mathrm{H}-$ $\mathrm{R}$ ) diagrams for stars of similar abundances.

A collection of young (non-cluster) A stars will usually be randomly distributed on and above the main-sequence in an $\mathrm{H}-\mathrm{R}$ diagram, because they are formed stochastically over time, and vary in the main-sequence ${ }^{2}$ ). than the younger stars.) colour and luminosity as they evolve, at different rates, off the main-sequence towards the red-giant branch. Blue stragglers, descendants of some of these stars, seem to remain in 'young' locations for longer than normal lifetimes, so they also show the random distribution of their progenitors, plus their own evolutionary peculiarities (there is evidence that they often appear just above, rather than on,

The new data on A stars show that the age distributions for normal young A stars near the galactic plane, and for distant high-velocity A stars, are completely different. Those within 500 parsecs of the plane are randomly distributed, as expected, from recently formed stars up to those aged $2.2 \times 10^{9}$ years.

In striking contrast, the ages of young $A$ stars at distances of more than 1 kiloparsec from the galactic plane are less than onethird of those observed for normal disk A stars - all are younger than $6.5 \pm 1.0 \times$ $10^{8}$ years old (Fig. 1). (This is not an effect of observational limits, because older A stars are intrinsically more luminous than main-sequence stars of the same colour, and if present, they would have been up to four times more likely to have been seen

Figure 2 shows the positions of some known blue stragglers ${ }^{4}$ in an $\mathrm{H}-\mathrm{R}$ diagram, compared with those of the distant (1-10 kiloparsecs) A stars. In contrast to the random locations of the blue stragglers, the A stars have the classical appearance of a group of stars formed within a relatively short period, evolving away from the main-sequence. To propose that the $\mathrm{A}$ stars are actually blue stragglers is to suggest that a disparate collection of

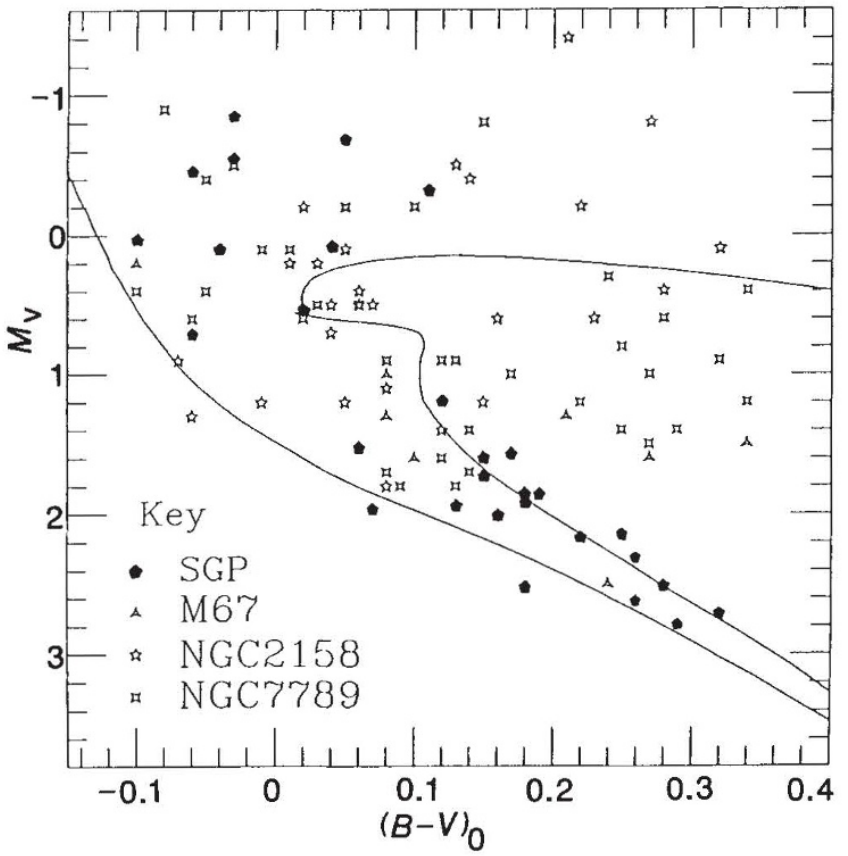

anomalous stars, in existence for thousands of millions of years, sampled over a large volume of the Galaxy, would fortuitously mimic the distribution of a young coeval group. It is not necessary for us fully to comprehend the mechanism of bluestraggler (or distant A-star) formation to appreciate that, according to a standard classification technique like the $\mathrm{H}-\mathrm{R}$ diagram, they are not the same kind of star.

Young A stars are seen far from the galactic plane in several directions ${ }^{3}$, which indicates that they are relatively widespread throughout our Galaxy. This distribution, plus their restricted age range, indicates that they originated from a single event that occurred on a galactic scale, such as the rapid accretion of a large quantity of gas, possibly from the merger of a satellite system with our Galaxy ${ }^{5}$. This would have resulted in the formation (from a mixture of accreted and disk gas) of high-velocity stars, at a specific epoch, with the unusual calcium abundances that are a feature of the distant young A stars ${ }^{2.3}$.

It remains to be seen whether this particular hypothesis is correct. On present evidence, it is clear that blue stragglers, or any other type of star formed randomly over time from normal galactic processes, cannot be proposed to explain the existence of young high-velocity A stars far from the galactic plane.

Catherine M. Lance Division of Radiophysics, CSIRO,

PO Box 76, Epping,

New South Wales 2121, Australia

1. Trimble, V. Nature 336. 111 (1988).

2. Lance. C.M. Astrophys. J. Suppl. Ser. 68, $463-485$ (1988)

3. Lance. C.M. Astrophys. J. 334, 927-946 (1988).

4. Hagen. G.L. Publ. David Dunlap Observ. 4, 3-62 (1970).

Rodgers, A.W.. Harding. P. \& Sadler, E. Astrophys. J. 244. $912-918$ (1981)

\section{More insect eating}

SIR-I would like to add some first-hand information to that provided by Sachi Sri Kantha on the subject of insectivory in Japan (Nature 336, 316; 1988).

As a child in the Nanshin district of the Fig. 2 The positions in an $\mathrm{H}-\mathrm{R}$ diagram of young South Galactic Pole A stars more than 1 kiloparsec from the plane, compared with some known blue stragglers from the clusters M67, NGC2158 and NGC7789. Isochrones are shown for the main-sequence and for stars $5 \times 10^{\circ}$ years old. southern Nagano prefecture in Japan, I consumed various species of insect. Many people living there still eat insects of ten subfamilies: Ephemeroptera (I cannot make further identification), Odonata (Libellulidae), Orthoptera (Tettigonioidea and Acridioidea), Plecoptera, Hemiptera (Cidadidae), Neuroptera, Tricoptera, Lepidoptera (pupae and adults of silk worm), Coleoptera (adults of true water beetles and water-scavenger beetles, plus larvae, pupae and adults of longicorn beetles) and Hymenoptera (all species of Vespa and Polistes inhabiting the district). In Nanshin supermarkets one can buy aquatic insects (canned), Vespa spp. (canned), rice hoppers and cooked pupae and adult silk worm. Most insects are cooked before eating but some people 